\title{
Jean-Michel Adam, Souvent textes varient, Paris, Classiques Garnier, 2018, 602 p.
}

Jean-Louis Lebrave

\section{(2) OpenEdition}

1 Journals

Édition électronique

URL : https://journals.openedition.org/genesis/3243

DOI : 10.4000/genesis.3243

ISSN : 2268-1590

Éditeur :

Presses universitaires de Paris Sorbonne (PUPS), Société internationale de génétique artistique littéraire et scientifique (SIGALES)

Édition imprimée

Date de publication : 15 décembre 2018

Pagination : 177-181

ISBN : 979-10-231-06282

ISSN : 1167-5101

\section{Référence électronique}

Jean-Louis Lebrave, « Jean-Michel Adam, Souvent textes varient, Paris, Classiques Garnier, 2018

602 p. », Genesis [En ligne], 47 | 2018, mis en ligne le 15 janvier 2019, consulté le 28 juin 2022. URL :

http://journals.openedition.org/genesis/3243 ; DOI : https://doi.org/10.4000/genesis.3243

Ce document a été généré automatiquement le 21 janvier 2021.

Tous droits réservés 


\title{
Jean-Michel Adam, Souvent textes varient, Paris, Classiques Garnier, 2018, 602 p.
}

\author{
Jean-Louis Lebrave
}

\section{RÉFÉRENCE}

Jean-Michel Adam, Souvent textes varient, Paris, Classiques Garnier, 2018, 602 p.

1 «Souvent textes varient » : aucun lecteur francophone ne peut manquer de répondre au puissant appel intertextuel que ce titre lance au premier hémistiche du célèbre vers attribué par Brantôme à François $\mathrm{I}^{\mathrm{er}}$. Mais c'est à une autre intertextualité que JeanMichel Adam fait d'abord référence, celle du titre d'un ouvrage du linguiste Jean Peytard ${ }^{1}$, dédicataire du livre, auquel il rend un hommage appuyé dans l'introduction (p. 50-54). "Souvent femme varie» est pourtant bien présent dans l'interdiscours conscient de l'ouvrage, comme on le découvre au détour du chapitre qui ouvre la deuxième partie du livre («Récrire les textes des autres»). Prenant son propre titre comme exemple de «l'émergence, dans le dire, du déjà dit d'un autre» (p. 214), Adam en explicite en effet le caractère «doublement dialogique». Double, car avant de revenir sur la "sémiotique de l'altération" de Peytard, qui a constitué "prioritairement» sa motivation, il discute de l'intertextualité première que le titre entretient « dans le thésaurus de la mémoire collective » avec Brantôme et François I ${ }^{\mathrm{er}}$. Adam signale d'abord la "misogynie déclarée » du vers complet, il affirme ensuite qu'en expulsant le mot, il a expulsé en même temps le contenu misogyne tout en en "gard[ant] la trace mémorielle», et il rappelle enfin sa dette théorique et intertextuelle envers Peytard (p. 215).

2 Les effets de sens produits par cette intertextualité affichée à propos de la variation sont un condensé saisissant des questions abordées dans cet ouvrage et des réponses qu'Adam leur apporte. À partir du constat premier que « les textes relevant du domaine 
de l'art verbal possèdent une capacité de résistance au temps et une plasticité » uniques dans l'ensemble des pratiques discursives (p.10), Adam pose l'existence d'une opération très générale de récriture ${ }^{2}$ - véritable fil rouge qui relie dans une théorie unifiée l'ensemble des études de cas qui composent le livre. C'est l'occasion pour lui de reprendre nombre de travaux antérieurs en les actualisant à la lumière de la bibliographie la plus récente et en les approfondissant, souvent d'une manière significative, pour mieux les intégrer à la stratégie globale de l'ouvrage. On peut d'ailleurs voir dans ce processus une forme éminente de l'intratextualité qui fait l'objet de la première partie.

3 Après une introduction substantielle dans laquelle Adam pose, à travers une discussion des notions de récriture, de série, de variation, de variance, les jalons de son parcours (p. 21-65), l'ouvrage enchaîne quatre parties, consacrées respectivement aux "variations génétiques et intratextuelles» (p.67-203), aux "variations intertextuelles» (p. 205-316), aux «variations éditoriales» (p. 317-423), et enfin aux «variations traductoriales» (p. 425-534). La conclusion (p. 535-551) plaide pour une interaction à (re)trouver entre les différentes disciplines du texte, elle fait l'éloge d'un " travail systématique et rigoureux sur les manuscrits, les éditions et les traductions [...] enrichi par un amour de la langue et des langues» (p.551), et souligne enfin la capacité de la linguistique à « de nouveau contribuer activement au développement des sciences littéraires»(ibid.). Malgré sa densité et son volume, le livre est d'une consultation très plaisante grâce à une bibliographie nourrie et à trois index détaillés des concepts, des auteurs, et des critiques.

4 Les positions théoriques d'Adam s'appuient toutes sur une impressionnante série d'observations qui témoignent à la fois d'une extrême rigueur dans la description des paramètres linguistiques et textuels, d'une profonde et riche culture textuelle, et d'un intense amour de la langue et de la littérature. Hugo (p. 79-100), Camus (p. 101-140 et 141-158), Duras (p. 159-203) viennent ainsi nourrir la problématique génétique de la première partie, et Desnos (p. 225-240), Cendrars (p.241-258), Char (p. 259-276), Perrault (p. 277-304) et Grimm réécrit par Beck (p. 305-316), les analyses intertextuelles de la deuxième, tandis que l'étude des variations éditoriales de la troisième partie se fonde sur des corpus empruntés à Baudelaire (p. 335-370) et Rimbaud (p. 371-424), et que la quatrième et dernière partie s'appuie sur un échantillonnage particulièrement riche des traductions de Die Verwandlung de Kafka (p. 443-484) et d'El Hacedor de Borges (p. 485-516), et sur une exploration des réflexions d'Artaud sur Lewis Carroll (p. 517-534).

5 Il n'est pas possible dans le cadre de ce compte rendu de reprendre toutes les études de cas du livre. Aussi me contenterai-je d'en illustrer l'extrême qualité par deux exemples, celui des Petits Poèmes en prose de Baudelaire et de «Phrases» de Rimbaud pour les variations éditoriales, et celui de Die Verwandlung de Kafka pour les variations traductoriales, avant de discuter pour finir, dans une perspective génétique, des implications de ses choix théoriques concernant l'intra-/intertextualité.

6 Commençons donc par la troisième partie, consacrée à la variation éditoriale. À vrai dire, cet intitulé pourrait prêter à confusion, car Adam vise les éditeurs scientifiques de textes, et non pas les phénomènes relevant de ce qu'on désigne depuis les travaux de Rudolf Mahrer sous le nom de génétique éditoriale. Les exemples de Baudelaire et de Rimbaud que Jean-Michel Adam commente portent sur la manière dont les textes des deux poètes ont été publiés par leurs éditeurs successifs; il pointe les 
incompréhensions dont ceux-ci se sont rendus coupables, et propose des façons de les corriger. De l'analyse consacrée aux Petits Poèmes en prose de Baudelaire, je retiendrai deux points. Le premier concerne les conditions éditoriales et matérielles qui ont donné naissance au genre du poème en prose. Comme Adam le montre d'une manière très convaincante, le poème en prose répond à une demande des éditeurs de journaux : fournir des textes susceptibles d'être publiés isolément tout en constituant une série au sein d'une suite de numéros consécutifs ou non; la contrainte supplémentaire à laquelle ces textes doivent satisfaire est de se plier aux exigences de mise en page des journaux. Ceci permet à Adam de poser que «la déclaration d'autonomie de chaque texte, qui leur confère un statut de fragments ou de morceaux interchangeables en fonction de la place disponible, est avant tout destinée à faciliter leur publication dans la presse » (p. 338). Adam s'inscrit par là dans la lignée de l'histoire du livre et de la bibliographie matérielle, auxquelles il consacre une synthèse dans l'introduction à cette troisième partie (p. 325-333).

7 Le second point est autant génétique que variationnel. Par une analyse linguistique et textuelle impressionnante de subtilité et de rigueur, Adam montre qu'un manuscrit de Baudelaire conservé à la Bibliothèque littéraire Jacques Doucet constitue l'avant-texte du poème en prose «La soupe et les nuages » et du poème "Les yeux de Berthe ", et il rapproche le motif du poète "mangeur de nuages ", présent dans le poème en prose, de celui du poète "prince des nuées" "exilé sur le sol " du poème "L'albatros", en dégageant ce qu'en référence à Saussure il appelle un "plexus de transpositions " (p. 367-368). Il en conclut que « la façon dont les vocables passent d'un texte à l'autre à l'occasion de reprises dans de nouvelles syntagmatiques et de nouvelles textualisations ouvre des lignes de variations qui sont autant de pistes de lecture » (p. 68), sans pour autant, précise-t-il, que cette chronologie dessine une genèse (p. 369).

Les Illuminations de Rimbaud permettent à Jean-Michel Adam d'approfondir sa savante dissection des variations éditoriales. L'examen attentif de l'origine du titre (p.372), celui des «manipulations » subies par le recueil du fait des éditeurs (p. 372), la prise en compte de l'évolution de l'écriture de Rimbaud (p.375), comme celle des valeurs sémantiques du mot barbare, titre par lequel se clôt le recueil dans le manuscrit autographe de Rimbaud conservé à la Bibliothèque nationale de France (p.376), l'amènent à proposer d'« éditer le recueil composé de la trentaine de poèmes numérotés de la main de Rimbaud, recueil dont le titre éditorial devrait être mise entre crochets [Illuminations] et ne pas comporter d'article, le déterminant "les" francisant l'anglais qu'on trouve par ailleurs dans le recueil» (p.379). Pour étayer encore davantage cette thèse, il s'appesantit sur le cas du poème intitulé «Phrases ». Par un examen très attentif des segmentations graphiques attestées dans le manuscrit, corrélé à une étude minutieuse du «travail de la phrase », il montre que « les traces graphiques fonctionnent comme autant d'instructions qui proposent au lecteur de fermer un tronçon de traitement pour en ouvrir un autre»(p.391). Soulignons la portée méthodologique de la démarche d'Adam, qui montre que, sous peine de ne rien comprendre au poème et de dénaturer la poétique de Rimbaud, un éditeur ne saurait faire l'économie d'une prise en compte des particularités matérielles (Louis Hay parlerait de sémiotique visuelle) de l'autographe et des caractéristiques linguistiques du texte. En guise de point final à ce trop rapide survol d'une investigation particulièrement fouillée, on donnera la citation suivante, qui synthétise l'apport de Jean-Michel Adam aux études textuelles : «Pour entrer dans le jeu poétique de poèmes en prose comme "Phrases", il faut accepter de partir de la structure textuelle de surface 
et de ses rythmes propres; il faut restituer le texte au langage au lieu de le fermer sur un référentiel biographique, historique ou ésotérique » (p. 421).

Placée sous le double patronage de Borges et de Meschonnic, la quatrième partie, consacrée au «bougé du texte et de la langue dans le traduire » se veut au carrefour de la linguistique, de la littérature et de la récriture définie comme une "expérience cognitive essentielle ». En même temps que ce bougé, Adam souligne «la persistance mémorielle du texte traduit dans la matérialité de son rythme, de ses répétitions et vocables et de sa syntaxe» (p. 431). Il pose que «traduire ne consiste pas seulement à faire passer des énoncés d'une langue dans une autre, mais à passer par une troisième langue : celle d'un texte, d'un poème, d'un livre ou d'un recueil» (p. 437), et pour lui « chaque traduction est [...] une lecture-écriture d'un texte dont le bouclage du sens ne saurait un jour être réalisé » (p. 440).

Ce n'est pourtant pas sous le signe d'une intertextualité positive, mais sous celui d'une trahison de l'original que se place l'étude des traductions de Die Verwandlung. Ainsi, Adam fait référence à Bernard Lortholary qui, dans la préface qu'il place en tête de sa traduction, écrit que "même si Kafka ne choisit pas le terme international d'origine grecque, mais son synonyme proprement allemand, le titre de La Métamorphose situe ce récit dans une longue et riche tradition mythologique, littéraire, et quasi universelle ». Adam condamne à ce propos la « récupération » du «paradigme intertextuel» de la métamorphose par les traducteurs, qui n'acceptent pas «la banalisation de la transformation de Gregor et l'humour noir qui traverse [le] récit» (p.449). Et il souligne que le mot Verwandlung "fait sens aussi par le mot qui est expulsé de la surface du discours et qui reste dans la mémoire de la langue, sous le texte » (p. 449).

La dérive de cette intertextualité "récupérée » est très générale, puisqu'elle est attestée dans presque toutes les traductions recensées par Adam. Faut-il pour autant souscrire à la conclusion qu'il en tire, lorsque critiquant le "sentiment général des éditeurs, traducteurs et lecteurs cultivés, déroutés par un retour à la langue et au texte de Kafka et par le surgissement possible d'un autre texte", il s'insurge contre la "superstition fixiste de l'œuvre d'art " sous-jacente à cette attitude? On pourrait en effet, d'une manière un peu provocatrice, tirer la conclusion inverse, et voir dans la lecture «infidèle» du titre original de Kafka une confirmation éclatante de la variabilité textuelle chère à l'auteur et du potentiel d'interprétation créative inhérent à toute traduction. Certes, dans le cas de la nouvelle de Kafka, la variation textuelle impliquée dans le titre produit un déplacement du sens tout à fait considérable, déplacement qui engage de proche en proche l'ensemble du texte. Le présupposé sousjacent à la condamnation de cet état de fait par Adam semble être qu'il juge un tel déplacement illicite. Mais ce présupposé reste implicite, et soulève une question beaucoup plus générale, qui engage l'appréciation qualitative des variations textuelles liées aux différentes traditions culturelles qui accueillent les traductions. Rappelons seulement à ce propos les débats qu'a suscités la proposition de remplacer plus-value par survaleur pour traduire le concept marxiste de Mehrwert, ou ceux qui ont accompagné les tentatives de retraduction en français des concepts psychanalytiques élaborés par Freud. Par une productivité de la variation textuelle qui, au fond, illustre à merveille les thèses d'Adam, les traducteurs « infidèles » de Marx ou de Freud, comme ceux de Die Verwandlung, n'ont pas obéi à une "superstition fixiste " portant sur le texte qu'ils traduisaient, mais au contraire activé l'incontrôlable variabilité de celui-ci mieux vaudrait dire sa mobilité, sa ductilité, sa capacité à rester lui-même en devenant 
irrémédiablement autre. Jean-Michel Adam aurait-il reculé face à une intertextualité " malmenante ", qu'il aurait récusée parce que son résultat lui apparaissait comme une trahison de l'original, et non comme un enrichissement par ajout de couches de sens nouvelles? A-t-il cédé un peu vite à la piètre opinion qu'il semble avoir des traducteurs en général, en affirmant que la plupart de ceux-ci, « soucieux d'éviter les "répétitions" lexicales, oublient que de telles "répétitions" sont, très souvent, des lieux forts du sens » ? Il est vrai que, comme Adam le souligne lui-même, « la présence en palimpseste de traductions antérieures»(p.407) qui se dégage de la mise en parallèle des différentes traductions ne peut que mettre en valeur le caractère lui aussi très répétitif des inexactitudes et déformations que les traducteurs infligent au texte ${ }^{3}$.

Toujours à propos de Die Verwandlung, il est particulièrement intéressant pour nous de revenir un instant sur l'éclairage qu'Adam donne, en point d'orgue à son travail sur les traductions, à une comparaison entre le début de la nouvelle publiée en 1915 du vivant de Kafka et un passage du manuscrit inachevé daté de 1906-1907 publié de manière posthume sous le titre de Hochzeitsvorbereitungen auf dem Lande. Il postule en effet une relation génétique entre les deux textes, le fragment posthume pouvant selon lui être considéré comme un avant-texte dont le début de Die Verwandlung est la récriture. Très convaincante, l'analyse d'Adam interprète celle-ci comme transformation d'un "récit autobiographique saturé de marques de première personne [...] et au présent » à un "récit en troisième personne [...] et verbes aux temps de la narration historique " (p. 481). Une autre récriture, opérée celle-là par Kafka sur le manuscrit du Château et attestée par une trace graphique, vient confirmer la pertinence de la mise en relation effectuée par Adam : on se souvient en effet que le roman a commencé par être un récit à la première personne, et que c'est après une trentaine de pages que Kafka a remplacé tous les « Ich » par « K. ».

On y verra aussi - et ce constat me ramène aux deux premières parties du livre, qui concernent le plus directement la critique génétique - une convergence entre deux méthodes dont il faudrait examiner les conséquences théoriques. Dans le cas de Die Verwandlung, Jean-Michel Adam construit un avant-texte à partir de comparaisons linguistiques serrées entre des fragments textuels, mais sans traces attestées autres qu'une « intratextualité » partagée et une datation chronologique probable. De même, dans l'analyse du poème "Saison des semailles. Le soir» de Victor Hugo (p. 79-99), Adam inclut deux passages de l'œuvre de Victor Hugo dans son corpus textuel: un extrait d'une lettre, tirée du "Reliquat du Rhin» et datée d'octobre 1840, et un fragment d'un pamphlet politique extrait de Napoléon le Petit publié à Bruxelles en 1852. Et il entrelace ce qu'il appelle « la genèse intratextuelle d'un motif récurrent » (p. 85), c'est-à-dire des hypothèses sur l'ordre de l'écriture des œuvres élaborées à partir seulement des propriétés textuelles de celles-ci, avec l'examen du dossier génétique proprement dit, qu'il "constitue de seconde main» (p. 80) à partir d'extraits d'un carnet et du manuscrit autographe des Chansons des rues et des bois conservés à la $\mathrm{BnF}^{4}$. Il fait en outre allusion à une double intertextualité avec une parabole de l'Évangile selon saint Matthieu et le premier chapitre de La Mare au diable, ainsi qu'à une « intertextualité pluri-sémiotique » (p. 79) avec une gravure de Holbein et le célèbre tableau de Millet. Il convient d'ailleurs pour finir que «la proximité étonnante de ces deux textes réside certainement plus dans l'interdiscours que Sand et Hugo ont en partage [...] que dans la reprise de Sand par Hugo ». 
14 Je ne reprends pas l'analyse elle-même, remarquable en tout point par sa rigueur et sa productivité. Je voudrais isoler une phrase significative à mes yeux de la volonté d'Adam de «tenir méthodologiquement ensemble génétique du manuscrit et génétique intratextuelle» (p. 80). À propos du vers «J'admire, assis sous un portail, » il reprend la notion de "scène d'énonciation » élaborée par Dominique Mainguenau, et affirme à propos de la localisation du sujet lyrique qu'" on ne peut s'empêcher de penser à la "nichée sous le portail" tirée d'un poème des Contemplations, et au poème des Chansons des rues et des bois qui précède directement "Saison des semailles. Le soir" : "L’Église" " (c'est moi qui souligne).

Or on peut dire que dès sa naissance, la critique génétique s'est fait une obligation positiviste, si l'on veut - de précisément s'empêcher de penser à tout l'intertexte présent autour de l'œuvre dont on étudie la genèse, de ne pas fonder la construction du dossier génétique sur des traces textuelles seules et de s'en tenir à la stricte observation de traces matérielles attestées. Ceci afin de se démarquer de la critique littéraire "traditionnelle», et notamment de la critique des sources. C'est par exemple la position de Daniel Ferrer qui défend, à partir d'exemples empruntés à Montesquieu et à Joyce, «l'intérêt du recours aux documents de genèse " pour suppléer "l'insuffisance pratique de la notion d'agrammaticalité » qui est selon Riffaterre l'indice permettant au lecteur de repérer l'intertextualités. Lors de sa propre discussion du concept d'intertextualité (p. 207 et suivantes), Adam fait d'ailleurs référence à ce texte de Ferrer au terme de son parcours critique du concept et avant d'en donner sa propre définition: "l'intertextualité sera considérée ici comme un phénomène linguistique dialogique qui fait qu'un énoncé récrit des énoncés empruntés à un autre texte, littéraire ou non » (p. 211).

16 La position classique de la critique génétique est résumée par l'échange suivant, tiré d'un entretien, lui aussi mentionné par Jean-Michel Adam, paru dans Genesis 30, et au cours duquel Pierre-Marc de Biasi et Anne Herschberg-Pierrot dialoguent avec Antoine Compagnon. « ANTOINE COMPAGNON : [...] Les philologues ne sont plus des sourciers. Ils ne recherchent plus les sources biographiques ou bibliographiques comme ils le faisaient naguère. - ANNE HERSCHBERG PIERROT : Mais des traces intertextuelles. A. C. : En effet » (p. 55).

Que signifie vraiment pour la critique génétique ce remplacement de la critique des sources par l'intertextualité ? Ou, pour le dire autrement: Par delà le plaisir que procure la lecture des démonstrations aussi brillantes que convaincantes de JeanMichel Adam, jusqu'où peut-on le suivre dans sa proposition méthodologique d'entrelacer étude génétique, mises en parallèle intratextuelles et rapprochements intertextuels sans trahir ce qui constitue peut-être la spécificité la plus radicale de la critique génétique?

Dans le cadre de ce compte rendu, la question ne peut qu'être mentionnée. En tout cas, le remarquable livre de Jean-Michel Adam oblige à la poser, et fournit un certain nombre d'éléments précieux pour une discussion future. 


\section{NOTES}

1. Jean Peytard, Souvent textes varient..., Cahiers du CRELEF, Besançon, 1993.

2. Jean-Michel Adam explicite au tout début de son livre les raisons qui l'ont amené à préférer ce terme à celui de réécriture (Avant-propos, note 3 p. 9).

3. On consultera avec profit la notice que Jean-Pierre Lefebvre a placée en tête de sa retraduction de Die Verwandlung dans la nouvelle Pléiade Kafka (sortie le 11 octobre 2018). Outre qu'elle enrichit notablement nos connaissances sur la genèse du texte, elle apporte un intéressant contrepoint aux thèses défendues par Jean-Michel Adam.

4. Jean-Michel Adam utilise une reproduction donnée dans l'édition de l'Imprimerie nationale des CEuvres complètes de Victor Hugo parue chez Albin Michel en 1933.

5. Daniel Ferrer, "Quelques remarques sur le couple intertextualité-genèse ", dans P. Gifford et M. Schmid (éd.), La Création en acte. Devenir de la critique génétique, Amsterdam-New York, Rodopi, 2007. Article accessible en ligne sur le site de l'ITEM.

\section{AUTEURS}

JEAN-LOUIS LEBRAVE

ITEM (CNRS-ENS) 